\title{
Ectopic Overexpression of Lectin in Transgenic Brassica juncea Plants Exhibit Resistance to Fungal Phytopathogen and Showed Alleviation to Salt and Drought Stress
}

\author{
Deepak Kumar ${ }^{1}$, Shashi Shekhar ${ }^{1}$, Sandeep Bisht ${ }^{2}$, Vivek Kumar ${ }^{3}$, Ajit Varma ${ }^{3}$ and Manoj Kumar ${ }^{3 *}$ \\ ${ }^{1}$ School of Life Sciences, Jawaharlal Nehru University, New Delhi-110067, India \\ ${ }^{2}$ Department of Molecular Biology and Biotechnology, VCSG college of Horticulture, Uttarakhand University of Horticulture and Forestry, Pauri-246123, Uttarakhand, India \\ ${ }^{3}$ Amity Institute of Microbial Technology, Amity University Uttar Pradesh, Noida-201303, India
}

\begin{abstract}
Lectins are ubiquitous in nature and one of the most important secondary metabolites in plants. They are heterogeneous class of proteins that bind specifically and reversibly to carbohydrates and are used as a defence tool against various abiotic and biotic stresses. In this study, we describe generation of transgenic Brassica juncea plants over-expressing chickpea lectin in conferring fungal resistance. In addition, the transgenic plants also showed resistance against salinity $(\mathrm{NaCl})$ and mannitol induced drought stress. The stable integration and transcript expression of the transgene was confirmed by southern blot and RT-PCR analysis in the transgenic plants. The transgenic lines were evaluated for resistance against Alternaria brassicae as assessed by the number and size of lesions and time taken for leaf necrosis, the Alternaria infection was delayed and restricted in the transgenic plants with the protection varying from $36-60 \%$ in different transgenic lines compared to the Untransformed Control (UC) plants. In addition, the transgenic Brassica juncea plants also showed tolerance against high salinity and drought stress. The Expression of lectin gene imparts greater water retention capacity, higher proline content and more chlorophyll retention with less membrane damage and reduction in electrolyte leakage in the transgenic plants compared to UC plants. Together, these findings suggest the potential use of chickpea lectin gene to engineer the crop plants for broad spectrum biotic and abiotic stress tolerance to improve crop productivity.
\end{abstract}

Keywords: Abiotic stress; Alternaria leaf spot; Brassica juncea; Lectin; Transgenic

\section{Introduction}

Agricultural plants face constant state of war against an array of pathogens and adverse environmental factors which are major causes in limiting crop production and have been responsible for scripting some of the most devastating famines in the human history. Brassica juncea belongs to the Cruciferae family and it is an important oil seed crop grown in many countries around the world. The oil obtained from the seeds is the main cooking medium in different parts of the world, especially in the Indian sub-continent. Brassica is also a high biomass crop and may facilitate phytoremediation of heavy metals in polluted soils [1]. In India, mustard contributes $28.6 \%$ to the total oilseed production and has been projected to provide for $41 \%$ (14 million tons) of the country's demand by the year 2020 [2]. Due to increased demand for edible oil and biofuels, the cultivation of oilseeds crops has gained significant importance [3]. Unfortunately, the productivity of this crop has been hampered by several biotic and abiotic factors [4]. Among biotic stress one of the major constraints affecting the productivity of mustard is the Alternaria leaf spot disease caused by Alternaria brassicae [5]. However, the crop is also severely affected by abiotic stresses resulting in significant loss in seed yield. Conventional breeding approaches to develop the resistant cultivars is confounded due to non availability of suitable resistance sources within the available germplasm of cultivated and wild species of Brassica. Therefore, better combat strategies relying on generation of transgenic plants armored with defense proteins are being explored [6].

The plants have inherent defence mechanisms and an innate immune system to protect themselves against a wide range of pathogens by the discrimination between self and non-self through pathogenassociated molecular patterns [7]. The preformed physical and chemical barrier constitutively produced antimicrobial compounds which protects the plants against biotic and abiotic stress. The proteins embedded in the cell wall and plasma membrane is also an important sentinel of the system for the recognition and transduction of defense associated signals [8]. The susceptibility of plants to the pathogens is resulted due to the disruption of cell wall-plasma membrane adhesion during pathogen penetration into host cells, causes reduction in cell wall-associated defense responses, thereby making the plant more susceptible to disease [9].

Lectins are the ubiquitous carbohydrate binding proteins possess at least one non-catalytic domain that specifically and reversibly bind to mono or oligosaccharide structures and mediate different biological processes [10]. Plants lectins is also believed to be tool in the specific protein-carbohydrate interactions within the cytoplasmic and nuclear compartments [11] and to the glycoproteins present on the cell surfaces [12]. Lectin shows high affinity binding with the carbohydrate moiety of glycoproteins, glycolipids, polysaccharides and typical carbohydrate constituent of fungal cell walls [13]. Expression of Gastrodia elata lectins resulted into strong induction of Trichoderma viride, which

*Corresponding author: Manoj Kumar, Amity Institute of Microbial Technology, Amity University Uttar Pradesh, Noida-201303, India, Tel: +91-120-4392432, E-mail: manojjnu@gmail.com

Received: March 16, 2015; Accepted: March 24, 2015; Published: March 31, 2015

Citation: Kumar D, Shekhar S, Bisht S, Kumar V, Varma A (2015) Ectopic Overexpression of Lectin in Transgenic Brassica juncea Plants Exhibit Resistance to Fungal Phytopathogen and Showed Alleviation to Salt and Drought Stress. J Bioengineer \& Biomedical Sci 5: 147. doi:10.4172/2155-9538.1000147

Copyright: ( 2015 Kumar D, et al. This is an open-access article distributed under the terms of the Creative Commons Attribution License, which permits unrestricted use, distribution, and reproduction in any medium, provided the original author and source are credited. 
Citation: Kumar D, Shekhar S, Bisht S, Kumar V, Varma A (2015) Ectopic Overexpression of Lectin in Transgenic Brassica juncea Plants Exhibit Resistance to Fungal Phytopathogen and Showed Alleviation to Salt and Drought Stress. J Bioengineer \& Biomedical Sci 5: 147. doi:10.4172/2155- 9538.1000147

Page 2 of 7

indiates that lectin is an important defence protein in plants [14]. By the incorporation of the precursor gene of stinging nettle isolectin I into tobacco, the spore germination of Botrytis cinerea, Colletotrichum lindemuthianum, and T. viride was significantly reduced [15]. Several other plant lectins are also reported for their crucial role in the defense against phytopathogenic infection such as the potato lectin immobilizes avirulent strains of Pseudomonas solanacearum on the plant cell wall; however, avirulent strain is not recognized by lectins [16]. Hevein, a type of lectin produced by rubber tree latex shows in vitro antifungal activity against Botrytis cinarae, Fusarium oxysporum, and Fusarium culmorum [17] and also confers the protection against Alternaria leaf spot in Indian mustard [18]. Different plant lectins show their differential expression under abiotic stress including drought, salinity and temperature shock [18-22] in the different crop plants.

In view of the diversity in the protective action of lectin, we assessed the efficacy of the chickpea lectin gene, which was involved in the suppression of Alternaria leaf spot in Brassica juncea during the fungal infection. Also, the transgenic plants harbouring chickpea lectin showed enhancement in the tolerance to salinity and drought stress in comparison to UC plants. Collectively, the results of our study demonstrated the current efforts to generate broad spectrum of transgenic plants resistance to fungal phytopathogens along with abiotic stresses.

\section{Materials and Methods}

\section{Plant material}

The seeds of Brassica juncea cv. Varuna were procured from Indian Agricultural Research Institute, New Delhi-12.

\section{Bacterial strain and plasmid}

The chickpea letin cloned at the EcoRI-XbaI site in the pRT101 vector in between CaMV $35 \mathrm{~S}$ promoter and polyA terminator sequences. This promoter-lectin cDNA-terminator cassette was released from pRT101 vector by digestion with HindIII and cloned at the HindIII site in the multiple cloning site of pCAMBIA 1301 (pC1301) vector to give rise to pCAM-lectin. pC1301 has hygromycin as plant selection marker and kanamycin as bacterial selection marker. This construct was confirmed by restriction digestion with HindIII as well as by PCR amplification of lectin sequence by using specific primers. Further the gene construct was mobilized into Agrobacterium tumefaciens strain GV 3101 by Freeze (Instead of heat) and thaw method. Agrobacterium culture was maintained on solid YEB medium and selected with $50 \mathrm{mgl}^{-1}$ kanamycin and $100 \mathrm{mgl}^{-1}$ each of rifampicin and gentamycin.

\section{Transformation, selection and plant regeneration}

The construct, pCAM-lectin, was mobilized into Agrobacterium tumefaciens strain GV 3101, which was subsequently used to transform hypocotyls explants from 5 day old seedlings of Brassica juncea (cv. Varuna). The explants were further plated on the selection medium, $\mathrm{MSB}_{1} \mathrm{~N}_{1}$ (MS medium supplemented with $1 \mathrm{mgl}^{-1}$ each of 6-benzylaminopurine and $\alpha$-naphthaleneacetic acid) containing $\mathrm{AgNO}_{3}\left(3.4 \mathrm{mgl}^{-1}\right)$ and hygromycin $\left(30 \mathrm{mgl}^{-1}\right)$. The plates with explants were kept in culture room maintained at $22 \pm 2^{\circ} \mathrm{C}$ and $14 \mathrm{~h}: 10 \mathrm{~h}$ (light: dark) photoperiod. The shoots obtained after 15-20 days were subcultured on the rooting medium MSI $_{2}$ (MS medium supplemented with $2 \mathrm{mgl}^{-1}$ Indole-3-butyric acid). The established untransformed control (UC) and putative transgenic plantlets were transferred to glass house to hardened and to grow under $22 \pm 2^{\circ} \mathrm{C}$ temperature under 14 h:10 h (light: dark) photoperiod, with 70\% relative humidity.

\section{DNA extraction and PCR Analysis of the Transgenic Plants}

Genomic DNA was extracted from the leaves of UC and transgenic (TR) plants following the protocol of [23]. The primary screening for the selection of putative transformants was performed by polymerase chain reaction (PCR) analysis using the lectin gene specific primers mentioned below: The primer set were designed from conserved region of lectin gene

\section{Forward: 5'-ATGCATCTGCAGCTTGCTTA- 3'}

\section{Reverse: 5'-TCGATTATGGCTTCTCTTCAAA-3'}

The PCR reaction was carried out with Taq DNA polymerase enzyme mix along with supplied Taq buffer. The $\mathrm{Mg}^{2+}$ concentration was $1.5 \mathrm{mM}$ in $50 \mu \mathrm{l}$ reaction volume. PCR conditions were 35 cycles of $1 \mathrm{~min}$ at $94^{\circ} \mathrm{C}, 1 \mathrm{~min}$ at $55^{\circ} \mathrm{C}$, and $1 \mathrm{~min}$ at $72^{\circ} \mathrm{C}$, using $1 \mu \mathrm{g} / \mu \mathrm{l}$ of the isolated DNA as template and 10 pmol of each primer in a $50-\mu l$ reaction. At the end of 35 cycles, the reactions were incubated at $72^{\circ} \mathrm{C}$ for $10 \mathrm{~min}$ and the tubes were kept at $4^{\circ} \mathrm{C}$. An aliquot of the PCR product was electrophoresed on a $1.2 \%$ agarose gel and visualized by ethidium bromide staining on UV Trans-illuminator.

\section{Southern blot analysis}

Ten micrograms of genomic DNA from UC and TR plants was digested with PstI restriction endonuclease, electrophoresed on a $0.8 \%$ agarose gel and transferred to a Hybond-Nylon membrane (Amersham Biosciences, UK) using the capillary transfer (35). The membrane was prehybridized for $2 \mathrm{~h}$ at $65^{\circ} \mathrm{C}$ in a buffer containing $0.5 \mathrm{M}$ sodium phosphate buffer, pH 7.2, 1 mM EDTA, and 7\% SDS. Thereafter the denatured radiolabeled probe (834 bp Lectin amplicon obtained by PCR amplification of lectin gene) labelled with $\left[\alpha-32_{\mathrm{p}}\right] \mathrm{dCTP}$ using Random primer DNA labeling kit (Amersham Biosciences, UK) as per the manufacturer's instructions) was added to the prehybridization buffer and incubated overnight at $65^{\circ} \mathrm{C}$. The membrane was washed $(10$ min per wash) sequentially in $3 \mathrm{X}$ SSC, $0.1 \%$ SDS; $0.5 \mathrm{X}$ SSC, $0.1 \%$ SDS; $0.2 \mathrm{X} \mathrm{SSC} ; 0.1 \%$ SDS with constant agitation at $65^{\circ} \mathrm{C}$. The hybridization signals were captured using phosphorimaging (FLA 5000 imaging system, Fujiflim).

\section{In Vivo plant bioassay for resistance to fungal phytopathogen}

The assessment of antifungal activity of lectin tin he UC and TR plants were assessed. Alternaria brassicae (Accession No. ITCC 5097) procured from the Division of Plant Pathology, Indian Agricultural Research Institute, New Delhi. The fungal culture was multiplied on potato dextrose agar (PDA) medium at $22 \pm 1^{\circ} \mathrm{C}$ for the maintenance. The spore suspension was prepared as explained by [24]. The spore suspension was filtered through cheesecloth to remove the mycelia debris. The spores were washed with sterile distilled water and resuspended to a count of $10^{3}$ spores $\mathrm{ml}^{-1}$. For in vivo plant bioassay, the fourth leaf (from the top) of the 45-day-old UC and TR plants were painted with the spore suspension using a painting brush. The inoculated plants were covered with polybag to maintain the humidity which was conducive to infection. To assess the disease severity the development of necrotic lesions in terms of number and area on the inoculated leaf of UC and TR plants were observed and recorded up to 15 days of inoculation. Percentage disease protection was calculated by [6].

Disease protection $(\%)=\{$ (No: of lesions per leaf in UC plant- No. of lesions per leaf in TR plants) $\} /\{$ (No: of lesions per leaf in UC plant) $\}$ $\times 100$ 
Citation: Kumar D, Shekhar S, Bisht S, Kumar V, Varma A (2015) Ectopic Overexpression of Lectin in Transgenic Brassica juncea Plants Exhibit Resistance to Fungal Phytopathogen and Showed Alleviation to Salt and Drought Stress. J Bioengineer \& Biomedical Sci 5: 147. doi:10.4172/2155- 9538.1000147

Page 3 of 7

\section{Stress tolerance experiments}

For drought and salt stress treatments 6-day-old seedlings of UC plantlets grown on MS plates and TR lines grown on MS plates supplemented with $30 \mathrm{mgl}^{-1}$ hygromycin were transferred into pots containing agropeat in a growth chamber at $22 \pm 2^{\circ} \mathrm{C}, 60 \%$ relative humidity, and $16 \mathrm{~h}: 8 \mathrm{~h}$ (Light:dark) photoperiod. To assess the drought and saline stress the UC and TR plants were irrigated biweekly with $300 \mathrm{mM}$ mannitol and $300 \mathrm{mM} \mathrm{NaCl}$ solution respectively followed by rewatered for 6 days to recovery. These plants were used for performing different experiments.

\section{Leaf disc senescence assay}

To analyse the preliminary stress responses the leaf disc senescence assay was performed. For leaf disc senescence assay, leaf sections of equal diameter were excised from the fourth leaf from top of UC and TR B. juncea plants and floated on liquid MS basal medium containing $\mathrm{NaCl}$ (200-600 mM) or Mannitol (200-600 mM). Chlorophyll content in the leaf sections was estimated after 5 days according to the procedure of [25].

\section{Measurement of electrolyte leakage}

The Electrolyte leakage was determined according to the method described by [26]. Leaves from UC and TR plants were harvested and washed with deionized water for three times to remove surfaced electrolytes. Further they were immersed to the $10 \mathrm{ml}$ of deionized water in the test tubes to measure the electrical conductivity (EC1). After incubation for $30 \mathrm{~min}$ at $55^{\circ} \mathrm{C}$ again EC2 was determined. The samples were heated for $1 \mathrm{~h}$ in boiling water before the total conductivity was measured in the solution (EC3). Relative ion leakage was expressed as a percentage of the total conductivity:

$$
\text { Relative Electrolyte Leakage }=(\mathrm{EC} 2-\mathrm{EC} 1) / \mathrm{EC} 3] \times 100
$$

\section{Measurement of MDA content}

The protocol of Heath and Packer was used to measure MDA content [27]. $1 \mathrm{gm}$ of leaves of UC and TR plants were grinded to fine powder in liquid nitrogen. $3 \mathrm{ml}$ of $10 \%$ trichloroacetic acid was added to $0.2 \mathrm{~g}$ of the homogenized powder and left at $4^{\circ} \mathrm{C}$ for overnight. Further the homozenized powder was centrifuged at $1,000 \times \mathrm{g}$ for 20 min and supernatant was transferred to a new tube. $2 \mathrm{ml}$ of $0.6 \%$ thiobarbituric acid (TBA) was added to $2 \mathrm{ml}$ of the supernatant. The mixture was vortexed thoroughly and heated in boiling water bath for $15 \mathrm{~min}$, cooled immediately and centrifuged. The Absorbance was taken at 532 and $450 \mathrm{~nm}$ by using water as blank. The formula for the calculation of MDA content was: MDA content was:

$$
\left(\mu \mathrm{moll}^{-1}\right)=6: 45 \times \mathrm{OD}_{532} \times 0: 56 \times \mathrm{OD}_{450}
$$

\section{Measurement of relative water content (RWC)}

To determine the RWC, fresh leaves were detached from the UC and TR plants and weighed their fresh weight followed by dipping them in distilled water for $12 \mathrm{~h}$. The leaves were blotted to wipe off excess water and weighed to record turgid weight (TW). Further leaves were placed at $70^{\circ} \mathrm{C}$ for $24 \mathrm{~h}$ to record the dry weight (DW). The RWC was determined by the equation:

$$
\text { RWC }=(F W-D W) \times 100 /(T W-D W) .
$$

\section{Estimation of proline}

Proline content was estimated using the standard protocol [28]. $100 \mathrm{mg}$ of leaf tissue from UC and TR plants were homogenized in 10 $\mathrm{ml}$ of $3 \%$ sulfosalicylic acid. The homogenate was centrifuged at 15,000 rpm. $2 \mathrm{ml}$ of the supernatant was mixed with $2 \mathrm{ml}$ of $0.2 \%$ ninhydrin and $2 \mathrm{ml}$ of glacial acetic acid. Further the mixture was incubated at $100^{\circ} \mathrm{C}$ for $1 \mathrm{~h}$ and the reaction was terminated on ice. Extraction of Proline was done by $4 \mathrm{ml}$ of toluene. The OD of the supernatant was measured at $520 \mathrm{~nm}$ and proline concentration was determined from the standard curve made by using the purified L-proline (Sigma, MO, USA) and calculated on a Fresh Weight (FW) basis $\left(\mu \mathrm{molg}^{-1}\right)$.

\section{Results}

Generation and molecular characterization of transgenic $B$. juncea expressing chickpea lectin gene

Generetaion of transgenic $B$. juncea harbouring chickpea lectin gene was achieved by Agrobacterium mediated genetic transformation. The plasmid, pCAMBIA1301, contains chickpea lectin gene cloned between HindIII restriction site with CaMV $35 \mathrm{~S}$ constitutive promoter and NOS termination sequence (Figure 1a). The hygromycin phosphotransferase (hpt) gene present in the plasmid was used for putative transformants selection. Preliminary screening for the transgenic B. juncea plants, selected on hygromycin was done by PCR amplification of the chickpea lectin gene. In the six independent transgenic lines, an amplicon of 834 bp was found with the chickpea lectin gene specific primer (Figure 1b). These fragments were not amplified in the UC plants. The six PCR positive TR lines produced southern positive bands with single (L1, L3, L5, L6) and double copy (L2, L4) of the transgene after the restriction digestion with PstI (Figure 1c). To check the expression level of the chickpea lectin gene in the southern positive transgenic lines, RT-PCR analysis was performed followed by the densitometrically normalizing the actin levels. Figure 1d shows the presence of 834 bp band corresponding to the lectin successfully expressed in all the confirmed transgenic lines whereas it was absent in untransformed control plant. All the transgenic plants were morphologically similar to the UC plants and showed no visible signs of impaired growth or physiology. Further these TR lines and the UC plants were used for the

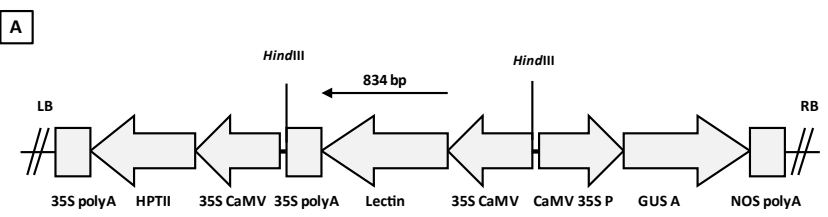

Figure 1a:


Figure 1b: Transformation and molecular analysis of Brassica juncea plants expressing lectin gene. (b) PCR analysis to screen the transgenic plants for the presence of transgene was done using lectin gene specific primers. 
Citation: Kumar D, Shekhar S, Bisht S, Kumar V, Varma A (2015) Ectopic Overexpression of Lectin in Transgenic Brassica juncea Plants Exhibit Resistance to Fungal Phytopathogen and Showed Alleviation to Salt and Drought Stress. J Bioengineer \& Biomedical Sci 5: 147. doi:10.4172/2155- 9538.1000147

Page 4 of 7

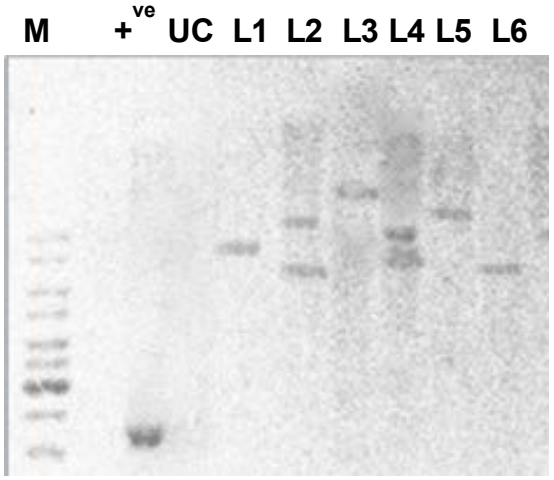

Figure 1c: Transformation and molecular analysis of Brassica juncea plants expressing lectin gene. (c) Southern blot analysis of the transgenic plants showing the integration and copy number of the transgene after digestion of genomic DNA with Pstl.

\section{UC $\quad$ L1 $\quad$ L2 $\quad$ L3 $\quad$ L4 $455 \quad$ L6}

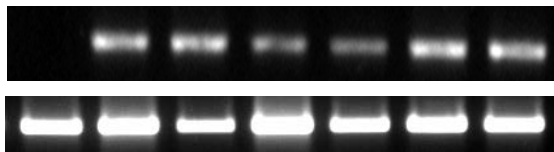

Figure 1d: Transformation and molecular analysis of Brassica juncea plants expressing lectin gene.. (d) RT-PCR analysis confirming expression of lectin in young fully expanded leaves of transgenic Brassica juncea plants. Actin gene was used as an internal control.

\begin{tabular}{|c|c|c|c|}
\hline Plants & $\begin{array}{c}\text { Number of lesions } \\
\text { (Avg } \mathbf{~ S D )}\end{array}$ & $\begin{array}{c}\text { Diameter range of } \\
\text { spots } \mathbf{( c m )}\end{array}$ & $\begin{array}{c}\text { Disease protection } \\
\text { (\% } \mathbf{\pm} \text { SD) }\end{array}$ \\
\hline UC & $14.0 \pm 1.3$ & $0.6-1.4$ & - \\
\hline L1 & $5.6 \pm 1.0$ & $0.3-0.6$ & $57.9 \pm 3.6$ \\
\hline L2 & $5.8 \pm 0.9$ & $0.3-0.7$ & $56.5 \pm 3.9$ \\
\hline L3 & $8.4 \pm 0.8$ & $0.4-0.9$ & $38.6 \pm 3.5$ \\
\hline L4 & $8.7 \pm 1.3$ & $0.2-1.0$ & $36.5 \pm 5.3$ \\
\hline L5 & $5.2 \pm 0.7$ & $0.2-0.5$ & $60.6 \pm 6.4$ \\
\hline L6 & $6.1 \pm 1.1$ & $0.3-0.8$ & $54.4 \pm 5.2$ \\
\hline
\end{tabular}

Table 1: Evaluation of Alternaria brassicae infection on $B$. juncea after 15 days of inoculation. Number of fungal lesions and the diameter range of lesions were determined. The percentage disease protection in the transgenic plants with respect to the Untransformed Control plants was determined as described in materials and methods. The data presented is the average \pm SD of three replicate experiments.

abiotic stress tolerance assay and in vivo anti fungal assay against the A. brassicae.

\section{In vivo biotic stress assay}

The resistant/tolerant efficiency of the candidate gene against the Alternaria leaf spot disaese was determined by In vivo plant bioassay, under controlled environmental condition. In the aforesaid protocol (Material and methods) the leaves of UC and the TR lines were inoculated with fungal spores. Further the initiation and progression of the disease lesions were analyzed in the subsequent days. After 15 days, the number of characteristic lesions on the leaves of transgenic plants was less ( $<9$ lesions/leaf) in comparison to the leaves of the UC plants ( $>14$ lesions/leaf) (Table 1). The size of the lesions ranged between 0.6 to $1.4 \mathrm{~cm}$ in diameter in the UC plants as against 0.2 to $1.0 \mathrm{~cm}$ in the different TR lines (Table 1). In the UC plants, the concentric rings of lesions were increasing in size until they merged with each other, which resulted into covering of whole lamina by the enlarged necrotic zone and the entire leaf dried up within 30 days of inoculation. The process of the spread of infection took more than 42 days in the case of TR plants. The percentage disease protection in the different TR lines compared with the UC plants varied between 36.6 to $60.6 \%$ (Table 1).

Transgenic plants over-expressing lectin showed higher chlorophyll retention, relative water content and proline accumulation with less membrane damage and electrolyte leakage under salt and drought stress

To investigate the effect of salt and drought stress at whole plant level, one month old plants of UCand the TR plants were transferred to the glass house in pots. To assess the effect of lectin expression on the salt and drought tolerance in transformed B. juncea. The one-monthold UC and TR plants were irrigated with $200 \mathrm{mM} \mathrm{NaCl}$ solution to induce salt stress and $200 \mathrm{mM}$ of mannitol solution to induce drought stress for 3 weeks and the performance of the UC and TR plants transgenic $B$. juncea plants were assessed by comparing few parameters that serves as important indicators of abiotic stress tolerance in plants.

Primarily the leaf disc senescence assay was performed to assess the ameliorative effects of lectin over-expression on leaf senescence and loss of chlorophyll due to salinity and drought stress (Figure 2a). There was loss of chlorophyll in UC plants leaf discs due to bleaching as compared to the transgenic plants leaf discs under high salt $(200-600 \mathrm{mM} \mathrm{NaCl})$ and Mannitol (200-600 mM) treatments $(P \geq 0.05)$. Where the UC leaf discs showed 86 and $69 \%$ decline in total chlorophyll content due to $600 \mathrm{mM} \mathrm{NaCl}$ and $600 \mathrm{mM}$ Mannitol treatments respectively. The\% decreases of chlorophyll content in the TR lines L1 and L5 were 57 and $62 \%$ due to imposition of salt stress, whereas 34 and $41 \%$ reduction in chlorophyll content was observed due to mannitol induced drought stress in the leaf discs excised from the L1 and L5 lines of transgenic plants respectively (Figure $2 \mathrm{~b}$ ).

Relative tolerance of UC and TR plants to the salinity and drought stresses was also assessed by comparing the electrolyte leakage from the leaf tissues of UC and transgenic plants. Under the normal condition the percentage of electrolyte leakage in the UC and TR plants has not been showed significant difference. However, percentage electrolyte leakage increased up to $71.7 \%$ in the UC plants as against an increase to 55.5 and $52.2 \%$ in the TR lines L1 and L5 respectively. The percentage of electrolyte leakage in the UC plants under mannitol induced drought stress was up to $66.1 \%$ whereas the elevation in the electrolytic leakage was 48.7 and $41.6 \%$ respectively in the L1 and L5 TR lines as compared to the UC plants (Figure 3a).

The membrane damage due to the lipid peroxidation can be assessed by monitoring the formation of (Thiobarbituric acid reactive substances) TBARS due to the decomposition of the polyunsaturated fatty acids (PUFAs), Which is an important component of membrane lipids. We measured cellular TBARS concentration as one of the first consequences of oxidative stress, and also as an indicator of cellular damage. The MDA content in the UC and transgenic lines did not change significantly prior to the stress treatments. However, there was a significant increase in the MDA content in the UC plants compared to TR lines under salt and drought stress. UC plants showed 1.68 and 1.80 fold increment in the accumulation of MDA under salinity stress, whereas the fold increase in MDA accumulation due to drought stress in the UC plants were 1.63 and 1.68 as compared to the TR lines $\mathrm{L} 1$ and L5 respectively (Figure $3 \mathrm{~b}$ ).

During this study the proline content was also measured under 
Citation: Kumar D, Shekhar S, Bisht S, Kumar V, Varma A (2015) Ectopic Overexpression of Lectin in Transgenic Brassica juncea Plants Exhibit Resistance to Fungal Phytopathogen and Showed Alleviation to Salt and Drought Stress. J Bioengineer \& Biomedical Sci 5: 147. doi:10.4172/2155- 9538.1000147

Page 5 of 7
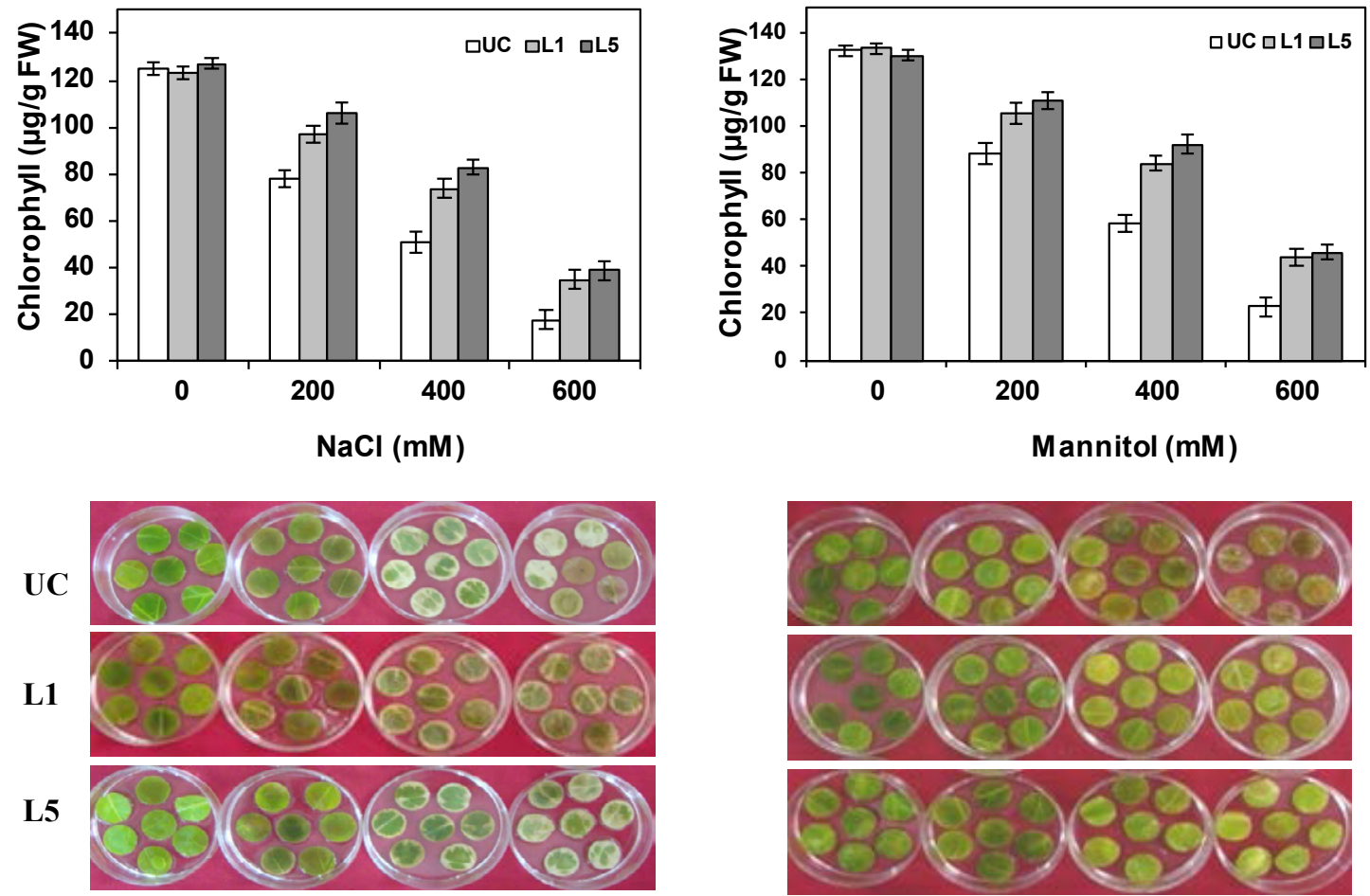

Figure 2: Leaf disc senescence under stress as measured by chlorophyll content. Chlorophyll content ( $\mu$ g/g fresh weight) of leaf discs from Untransformed Control (UC) and transgenic (line L1 and L5) Brassica juncea plants, kept for four days at different concentrations of $\mathrm{NaCl}$ (panel a), mannitol (panel b). The values are means \pm SE from three independent experiments. The photographs of UC and transgenic leaf discs under the corresponding treatments are presented below each plot.

the salinity and drought stress conditions. The TR lines L1 and L5 showed 3.2 and 3.4 times more proline accumulation respectively in comparison to the UC plants under saline stress, whereas the mannitol induced drought condition resulted into the 3.4 and 3.6 times higher proline accumulation in the TR lines L1 and L5 respectively in compared to UC plants (Figure 3c).

RWC is a parameter often used to assess the water retention capacity of plants. We assayed RWC in both UC and TR plants under non-stress and stress conditions. The RWC of UC plants was comparatively less as compared to the TR plants under drought and salt stress conditions. The RWC in leaf tissues of UC plants was reduced from $90 \%$ under nonstress to $48 \%$ and $56 \%$ in salt and drought stress conditions respectively whereas the decrease in RWC in the TR plants was from a control value of 90 and $91 \%$ (in lines L1 and L5) to 74\% and 76\% under salt stress and $71 \%$ and $74 \%$ under drought stress respectively (Figure $3 \mathrm{~d}$ ).

\section{Discussion}

The present study describes the introduction and expression of chickpea Lectin gene from into a widely grown cultivar of Indian mustard. The stringently transgenic plants were analysed through Southern blot analysis, which demonstrated that the transgenic lines were mostly containing a single copy of the transgene. The differential transcript expression profile of lectin observed between the different transgenic plants may be due to position effects of the transgene integration as the transgenes has integrated at different sites on the plant genome [29]. It is therefore, mostly desired to have single copy of candidate gene in transgenic lines.

Yield losses due to different abiotic and biotic stress are a serious problem in the cultivation of this crop [6]. The antifungal role of plant lectins due to their affinities to the carbohydrate are well documented in the scientific findings against several plant fungal phytopathogens $[17,18]$. The number and the size of lesions are important determiners for the degree of resistance to the leaf blight disease in Brassica species [24]. It is also reported the disease severity of Alternaria leaf blight caused by A. brassicae on the basis of the development of new lesions along with their expansion rate in terms of area and dimension in $\mathrm{B}$. Juncea plant [31] the present study the severity of disease in the terms of number and area of lesion were assessed. Fewer numbers of lesions were recorded on the leaves of TR lines as compared to the UC plants after the fungal infection caused by $A$. brassicae. Further the expansion rate of lesions was higher in the UC plants with respect to the transgenic lines. Srivastava and Srivastava reported that $A$. brassicae produces polygalacturonase and cellulases to degrade the cell wall of host plants [30]. It is well documented fact of research findings about the high avidity binding of lectins with the carbohydrate ligands present on the microbial surfaces. This high avidity binding causes the activation of opsonisation processes by the lectins by which the lectins interact with the phagocytic cells membrane receptors which lead to the stimulation of immune response. In view of the above findings, it can be conclude that, due to the interaction between the lectin and carbohydrates moiety on the fungal wall which resulted into the hypersensitive responses and opsonization which causes to the restriction of new lesion formation and reduction in the lesion area expansion on the leaves of transgenic plants compared to the UC plants. Reduction in the number and area of necrotic lesions are important marker of disease resistance in case of fungal infection in plant species.

Production of reactive oxygen species (ROS) is one of important manifestation of the abiotic and biotic stress conditions. To minimize the ameliorative effect of the ROS toxic metabolite, the plant produces 
Citation: Kumar D, Shekhar S, Bisht S, Kumar V, Varma A (2015) Ectopic Overexpression of Lectin in Transgenic Brassica juncea Plants Exhibit Resistance to Fungal Phytopathogen and Showed Alleviation to Salt and Drought Stress. J Bioengineer \& Biomedical Sci 5: 147. doi:10.4172/2155- 9538.1000147
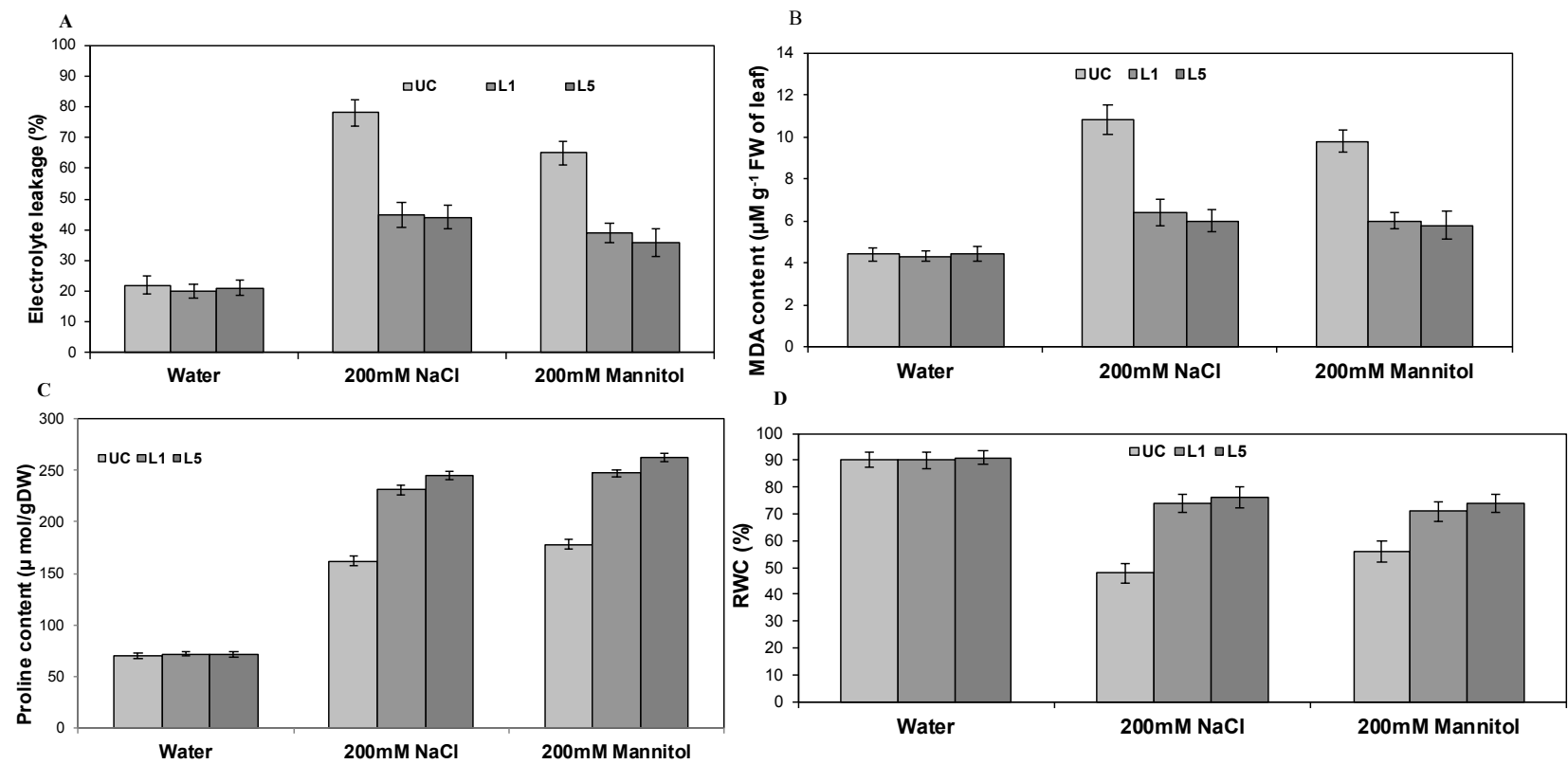

Figure 3: Comparison of abiotic stress tolerance of transgenic expressing lectin gene and Untransformed Control (UC) Brassica juncea plants. (a) Changes in electrolyte leakage from Brassica juncea leaf tissue as a percentage of total electrolytes in the leaves (b) MDA content (c) Proline content and (d) Relative Water Content (RWC) content in UC and transgenic plants exposed to $\mathrm{NaCl}(200 \mathrm{mM})$ or mannitol $(200 \mathrm{mM})$ stress. The plants irrigated with water served as the untreated control. Values are mean $\pm \mathrm{SD}$ of three experiments (three replicates per experiment). Results are significant at $P<0.05$.

different non-enzymatic effecter molecules. Relative tolerance of UC and TR plants to these stresses was assessed by measuring different parameters viz., electrolyte leakage, RWC, accumulation of MDA and proline [33]. Maintenance of high water content is an important marker of stress tolerance in plants. The results of the present study showed that RWC in TR lines L1 and L5 maintained at significant higher levels as compared to UC plants where it is decline due to imposed stress conditions. Bhushan et al. reported that lectin was expressed under stress condition and may be involved in the stabilization of the cytoskeleton structure under stress conditions [34]. Coefficient of lectin activity was increased by 2-15 times during the high temperature, osmotic and salinity stress in oil seed flax. The development of resistance by plants against abiotic stresses depends on the location of lectins in cell organelles. During the adaptation to stress the specificity of cytosolic and vacuolar lectins for carbohydrate are changed, such as total soluble lectin exhibit capacity to binding mannose and xylose, to saline stress galactose, glucose, and glucosamine. The candidate gene chickpea lectin was cloned under the CaMV 35 S promoter which constitutively expresses the lectin which was evident with the differential transcript expression. The constitutive expression of lectin might be responsible for maintenance of the cellular pool of lectin which could be regarded as the suppressor of the stress response by direct participation with modification in their quantitative and qualitative characteristics. Moreover it can be suggested that, the lectins may be regarded as drivers for plants to adapt various abiotic stresses. The increased electrolyte leakage under stress conditions in case of UC plants suggested much severe membrane damage compared to TR lines. The difference in the membrane damage was also evident in the higher MDA content in UC in comparison to TR lines. MDA accumulates as a result of lipid peroxidation, which is an effective indicator of oxidative damage. Therefore, it may be concluded that, lowering in the MDA content in the transgenic lines is resulted due to expression of lectin which is responsible for more stability of the cellular cytoskeleton structure in transgenic lines as compared to the untransformed control plants.
Our analyses also shows that lectin genes have been involved in biotic/ abiotic stress regulations and tandem/ segmental duplications may be regarded as drivers for plants to adapt various environmental stresses through duplication followed by expression divergence.

In conclusion, our work demonstrates the generation of an economically important oilseed crop Brassica juncea having broad spectrum resistance against fungal pathogens and in conferring tolerance against salinity and drought stress by transgenic expression of chickpea lectin. This could be a promising candidate to reduce the yield loss caused by the salinity, drought and Alternaria leaf blight disease and also serve as an alternate option for crop management and sustainable agriculture.

\section{Acknowledgment}

The authors are thankful to the Defence Research Development Organization (Project No.TC/2519/INM/-04/2012/CARS of INM1.2/311), Govt. of India for the partial financial support. The authors (particularly Manoj Kumar, Ajit Varma and Vivek Kumar) are thankful to Amity University, Noida and Lovely Professional University for facility and other administrative support.

\section{References}

1. Ebbs SD, Kochian LV (1998) Phytoextraction of zinc by oat (Avena sativa) barley (Hordeum vulgare), and Indian mustard (Brassica juncea). Environ Sci Technol 32: 802 -806.

2. Shekhawat K, Rathore SS, Premi OP, Kandpal BK, Chauhan JS (2012) Advances in agronomic management of Indian mustard (Brassica juncea (L.) Czernj. Cosson): An overview. International Journal of Agronomy.

3. Kumar PR (1999) Rapeseed mustard research in India: 21st century strategies. $10^{\text {th }}$ International Rapeseed Congress, Canberra, Australia.

4. Grover A, Pental D (2003) Breeding objectives and requirements for producing transgenics for major field crops of India. Curr Sci 84: 310-320.

5. Meena PD, Awasthi RP, Chattopadhyay C, Kolte SJ, Kumar A (2010) Alternaria blight: a chronic disease in rapeseed-mustard. Journal of Oilseed Brassica 1: 1-11.

6. Mondal KK, Bhattacharya RC, Koundal KR, Chatterjee SC (2007) Transgenic Indian mustard (Brassica juncea) expressing tomato glucanase leads to 
Citation: Kumar D, Shekhar S, Bisht S, Kumar V, Varma A (2015) Ectopic Overexpression of Lectin in Transgenic Brassica juncea Plants Exhibit Resistance to Fungal Phytopathogen and Showed Alleviation to Salt and Drought Stress. J Bioengineer \& Biomedical Sci 5: 147. doi:10.4172/2155- 9538.1000147

arrested growth of Alternaria brassicae. Plant Cell Reports 26: 247-252.

7. Jones JD, Dangl JL (2006) The plant immune system. Nature 444: 323-329.

8. Brugger AG, Lamotte O, Vandelle E, Bourque S, Lecourieux D, et al. (2006) Early Signaling Events Induced by Elicitors of Plant Defenses. Mol Plant Microbe Interact 19: 711-724.

9. Mellersh DG, Heath MC (2001) Plasma membrane-cell wall adhesion is required for expression of plant defense responses during fungal penetration. Plant Cell 13: 413-424.

10. Sharon N, Lis H (2004) History of lectins: From hemagglutinins to biological recognition molecules. Glycobiology 14: 53-62.

11. Damme EJ, Barre A, Rouge P, Peumans WJ (2004) Cytoplasmic/nuclear plant lectins: A new story. Trends Plant Sci 9: 484-489.

12. Hwang IS, Hwang BK (2011) The Pepper Mannose-Binding Lectin Gene CaMBL1 Is Required to Regulate Cell Death and Defense Responses to Microbial Pathogens. Plant Physiology 155: 447-463.

13. Broekaert WF, Parijs VJ, Leyns F, Joos H, Peumans WJ (1989) A chitin binding lectin from stinging nettle rhizomes with antifungal properties. Science 245 : 1100-1102.

14. Wang XC, Bauw G, Van Damme EJM, Peumans WJ, Chen ZL, et al. (2001) Gastrodianin-like mannose binding proteins: a novel class of plant proteins with antifungal properties. Plant J 25: 651-661.

15. Does MP, Houterman PM, Dekker HL, Cornelissen BJ (1999) Processing, targeting, and antifungal activity of stinging nettle agglutinin in transgenic tobacco. Plant Physiol 120: 421-432.

16. Parijs JV, Broekaert WF, Goldstein IJ, Peumans WJ (1999) Hevein: an antifungal protein from rubber-tree (Hevea brasiliensis) latex. Planta 183: 258-264.

17. Kanrar S, Venkateswari JC, Pulugurtha BK, Chopra VL (2002) Transgenic expression of hevein, the rubber tree lectin, in Indian mustard confers protection against Alternaria brassicae. Plant Science 162: 441-448.

18. Zhang W, Peumans WJ, Barre A, Astoul CH, Rovira P, et al. (2000) Isolation and characterization of a jacalin related mannose binding lectin from salt stressed rice (Oryza sativa) plants. Planta 210: 970-978.

19. Hirano K, Teraoka T, Yamanaka H, Harashima A, Kunisaki A, et al. (2000) Novel mannose binding rice lectin compose of some isolectins and its relation to a stress inducible salt gene. Plant Cell Physiol 41: 258-267.

20. Singh PS, Bhaglal P, Bhullar SS (2000) Wheat germ agglutinin (WGA) gene expression and $A B A$ accumulation in the developing embryos of wheat (Triticum aestivum) in response to drought. Plant Growth Regul 30: 145-150.
21. Shakirova FM, Bezrukova MV Shayakhmetov IF (1996) Effect of heat shock on dynamics of ABA and WGA accumulation in wheat cell culture. Plant Growth Regul 19: 85-87.

22. Murray MG, Thompson WF (1980) Rapid isolation of high molecular weight plant DNA. Nucleic Acids Res 8: 4321-4325.

23. Sharma TR, Singh BM, (1993) Parameters of resistance to Alternaria brassicae in some Brassica species, SABRAO Journal 25: 71-76.

24. Arnon DJ (1949) Copper enzymes in isolated chloroplasts: Polyphenoloxidase in Beta vulgaris. Plant Physiology 24: 1-15.

25. Sairam RK, Srivastava GC (2002) Changes in antioxidant activity in subcellular fractions of tolerant and susceptible wheat genotypes in response to long term salt stress. Plant Science 162: 897-904.

26. Heath RL, Packer L (1968) Photoperoxidation in isolated chloroplasts. I. Kinetics and stoichiometry of fatty acid peroxidation. Arch Biochem Biophys 125:189- 198

27. Bates LS, Waldren RP, Teare ID (1973) Rapid determination of free proline for water stress studies. Plant and Soil 39: 205-207.

28. Finnegan J, McElroy D (1994) Transgene inactivation: Plants fight back Biotechnology 12: 883-888.

29. Srivastava SK, Srivastava AK (1982) Polygalacturonase and cellulose production by Alternaria brassicae (Berk) Sacc. Var. Sarson. New Bot 9: 9-11.

30. Kuhlman M, Joiner K, Ezekowitz RA (1989) The human mannose-binding protein functions as an opsonin. J Exp Med 169: 1733-1745.

31. Rustagi A, Kumar D, Shekhar S, Yusuf MA, Misra S, Sarin NB (2014) Transgenic Brassica juncea plants expressing MsrA1, a Synthetic Cationic Antimicrobial Peptide, Exhibit Resistance to Fungal Phytopathogens. Molecular Biotechnology, 56: 535-545.

32. Kumar D, Yusuf MA, Singh $P$, Sardar M, Sarin NB (2013) Modulation of antioxidant machinery in $\alpha$-tocopherol-enriched transgenic Brassica juncea plants tolerant to abiotic stress conditions. Protoplasma 250: 1079-1089.

33. Bhushan D, Pandey A, Choudhary MK, Datta A, Chakraborty S, et al. (2007) Comparative Proteomics Analysis of Differentially Expressed Proteins in Chickpea Extracellular Matrix during Dehydration Stress. Mol Cellular Proteomics 6: 1868-84.

34. Levchuk AN, Voitovich EN, Lyakh VA (2013) Lectins of OilSeed Flax Plants Exposed to Abiotic Stress. Russian Journal of Plant Physiology 60: 77-83.

35. Sambrook J, Fritsch EF, Maniatis T (1989) Molecular cloning. A laboratory manual. New York: Cold Spring Harbor 